\title{
Proposta de Arquitetura para Controle Distribuído de Sistemas a Eventos Discretos Multiagentes Autônomos
}

\author{
Renan Sebem* André Bittencourt Leal* \\ * Programa de Pós-Graduação em Engenharia Elétrica, Universidade \\ do Estado de Santa Catarina, SC, (e-mail: renan.sebem@udesc.br).
}

\begin{abstract}
In this paper is proposed a distributed control architecture for autonomous multiagent discrete events systems. Each agent is composed by a local plant, a local controller and an interaction controller, all modelled as automata. Communication between agents are based in the extern events deactivation. Agents are considered to be identical. Considering the current state, agents only send the information of the events to be deactivated by others, ensuring safety through information privacy. Task disputes between agents are handled. The controller synthesis is proposed to be executed dynamically and embedded in the agent. The synthesis of a monolithic controller is not needed in this proposal. The proposed architecture allows the number of agents and the control specification to be variable through time, without the need of reconfiguration by the designer.

Resumo: Neste trabalho é proposta uma arquitetura de controle distribuído para sistemas a eventos discretos multiagentes autônomos. Nessa estrutura, cada agente é composto por uma planta local, um controlador local e um controlador de interação, os quais são modelados por autômatos. A comunicação entre os agentes é baseada na desabilitação de eventos alheios. Considera-se que os agentes são idênticos e que, em função do estado atual, cada agente envia aos demais os eventos que devem ser desabilitados por esses, garantindo a segurança através da privacidade da informação. Nesta proposta é desnecessária a síntese de um controlador monolítico e o cálculo do controlador para cada agente é realizado de maneira dinâmica e embarcada. A arquitetura proposta permite que tanto o número de agentes quanto as especificações de controle dos agentes sejam alterados ao longo do tempo de execução, tornando o controle adaptativo, sem que haja a necessidade de reconfiguração pelo projetista.
\end{abstract}

Keywords: Distributed Control, Multi-Agent Systems, Discrete Event Systems, Autonomous Systems, Autonomous Vehicles.

Palavras-chaves: Controle Distribuído, Sistemas Multiagentes, Sistemas a Eventos Discretos, Sistemas Autônomos, Veículos Autônomos.

\section{INTRODUÇÃO}

Há uma tendência de modularização de sistemas de engenharia de larga escala. Em sistemas massivos, tais como internet e trânsito de veículos, existem milhões de subsistemas que estão sujeitos a uma interação entre os próprios subsistemas. Esses sistemas são chamados de multiagentes. No caso da internet, os computadores são agentes que disputam por tarefas, tais como o acesso a um servidor, ou então disputam por velocidade de download através de um roteador. No caso do trânsito, os veículos são agentes que disputam pela rota que os levará aos seus destinos.

Quando se trata do controle de processos industriais, a aplicação do controle de sistemas multiagentes é adequada às tecnologias que consistem na base da quarta revolução industrial (chamada de indústria 4.0), como a Internet Industrial das Coisas (IIoT). Exemplos de aplicação utilizando tecnologias que estão relacionados com este trabalho são encontrados nos trabalhos de Kovalenko et al. (2019) e Gonzalez et al. (2018).
Dada a natureza distribuída de sistemas multiagentes é pertinente que o controle desses sistemas também seja distribuído. Ainda, os problemas citados a seguir inviabilizam a realização de um controle centralizado. O primeiro problema é que à medida que o número de agentes cresce, o tamanho do controlador centralizado cresce exponencialmente em número de estados. Este problema é resolvido na literatura pela modularização do sistema (de Queiroz and Cury, 2000), e também através da localização do supervisor (Cai and Wonham, 2010). Quando um sistema multiagente é modelado através destas técnicas, é necessário que o número de agentes seja fixo e que a especificação de controle não seja alterada ao longo do tempo. Outra maneira de resolver este problema é através do cálculo online do supervisor utilizando os modelos truncados do sistema, isto é, periodicamente apenas uma parte do supervisor é calculada. Um exemplo da aplicação desta técnica pode ser encontrado no trabalho de Tatsumoto et al. (2018).

Um problema ainda existente no estado da arte no controle distribuído de sistemas a eventos discretos multiagentes 
é a necessidade do cálculo de um supervisor monolítico na etapa de projeto. A solução deste problema não é o objetivo dos trabalhos mais recentes sobre o controle de sistemas multiagentes no âmbito da Teoria de Controle Supervisório (TCS) (Tatsumoto et al., 2018; Liu et al., 2018, 2019).

Ainda, nos trabalhos de Liu et al. (2018, 2019), após o cálculo do supervisor monolítico é realizada a localização (Cai and Wonham, 2010), ou seja, a distribuição dos supervisores locais. Este método pode se tornar inviável devido a explosão exponencial do número de estados em relação ao número de agentes. Porém, são exploradas as similaridades entre agentes para evitar a explosão exponencial de estados, calculando-se assim um supervisor monolítico chamado de escalável. No trabalho de Tatsumoto et al. (2018) o supervisor monolítico é calculado online, tornando possível o calculo com os modelos truncados do sistema evitando a explosão exponencial de estados. Embora a explosão exponencial de estados seja tratada nesses trabalhos, é importante destacar que os métodos utilizados ainda realizam o cálculo do supervisor monolítico.

Outro problema é que o número de agentes nos sistemas multiagentes pode ser variável (e.g. novos computadores podem ser conectados à internet a qualquer momento), e assim o controle deve ser recalculado cada vez que o número de agentes for modificado. Este problema é tratado em Liu et al. (2018, 2019), através do uso de supervisores escaláveis.

Avançando ainda mais no quesito do controle de sistemas multiagentes, um problema ainda não tratado na literatura é quando a especificação de controle é alterada durante a execução do sistema. Isto é, todos os trabalhos estudados até o momento consideram que a especificação de controle é fixa e não pode mudar em tempo de execução. Nas soluções atuais, a alteração da especificação de controle acarreta no reprojeto dos controladores, sendo necessária a intervenção do projetista.

A solução proposta nesses trabalhos considera que os agentes podem ter acesso a todas as informações de todos os agentes. Ou seja, é considerado que todos os agentes possuem um objetivo em comum. Essas soluções não contemplam o caso em que os agentes são concorrentes. Isto é, essas soluções não seriam seguras para o caso em que os agentes são concorrentes, pois eles teriam acesso às informações de outros agentes.

A arquitetura proposta neste trabalho permite que o controle seja projetado de maneira distribuída, i.e., em nenhuma etapa do projeto, nem de execução do controle, é necessária a obtenção de um modelo global que represente o comportamento global do sistema, evitando a explosão exponencial de estados. Ainda, permite que o número de agentes e as especificações de controle de cada agente sejam modificadas ao longo do tempo, sem a necessidade do recálculo dos controladores de todos os agentes. Fica evidente que, ao atingir esses objetivos, é dispensável a necessidade de um coordenador de tarefas. E, finalmente, a proposta contempla uma solução segura para o caso em que os agentes são concorrentes, ou seja, é fornecido o mínimo necessário de informação entre agentes.

\section{PRELIMINARES}

O conjunto finito de estados é denotado por $\Sigma$. $\Sigma$ pode ser particionado como $\Sigma_{o} \cup \Sigma_{u o}$, e também como $\Sigma_{c} \dot{\cup} \Sigma_{u c}$, onde $\Sigma_{o}, \Sigma_{u o}, \Sigma_{c}$ e $\Sigma_{u c}$ denotam os eventos observáveis, não observáveis, controláveis e não controláveis respectivamente. Uma string é uma sequência de tamanho finita de eventos pertencentes à $\Sigma$. O conjunto de todas as strings formadas por eventos em $\Sigma$ é denotado por $\Sigma^{*}$. Qualquer subconjunto de $\Sigma^{*}$ é denominado como linguagem sobre $\Sigma$. Considerando $L$ como uma linguagem sobre $\Sigma$. O prefixofechamento de $L$ é denotado por $\bar{L}$. Dada uma string $s \in L, P_{i}(s)$ é a projeção de $s$ sobre um alfabeto $\Sigma_{i}$, ou seja, os eventos que não pertencem à $\Sigma_{i}$ são removidos de $s$. Um autômato é definido como uma sêxtupla $G=$ $\left(Q, \Sigma, \delta, \Gamma, q_{0}, Q_{m}\right)$, onde $Q$ é o conjunto finito de estados; $\Sigma$ é o conjunto finito de eventos; $\delta: Q \times \Sigma \rightarrow Q$ é a função de transição; $\Gamma$ é a função de evento ativo; $q_{0}$ é o estado inicial; e $Q_{m}$ é o conjunto finito de estados marcados. A linguagem de um autômato é denotada por $L(G)$, tal que $L(G)=\left\{s \in \Sigma^{*}: \delta\left(q_{0}, s\right)\right.$ is defined $\}$. A linguagem marcada de um autômato é denotada por $L_{m}(G)$, tal que $L_{m}(G)=\left\{s \in L(G): \delta\left(q_{0}, s\right) \in Q_{m}\right\}$. Um autômato é dito não bloqueante se $\overline{L_{m}(G)}=L(G)$. Dados dois agentes identificados pelas letras $i$ e $j$, o estado atual de cada agente é determinado como $q_{i}$ e $q_{j}$, respectivamente. O conjunto de estados formado pelos estados atuais de todos os agentes é $\chi=\left\{q_{i}, q_{j}, \cdots\right\}$.

\section{ARQUITETURA PROPOSTA}

Nesta seção é descrita a proposta de arquitetura de controle para sistemas a eventos discretos multiagentes. A ocorrência de eventos é considerada instantânea no tempo, portanto dois eventos diferentes não ocorrem ao mesmo tempo.

O sistema como um todo é composto por múltiplos agentes, que são diferenciados através de um índice único para cada agente. Cada agente é composto pela planta local $G_{l o c}$, pelo controlador local $C_{l o c}$ e pelo controlador de interação $C_{\text {inter. }}$ A planta $G_{l o c}$ é representada pelo modelo em autômato que contém todos os possíveis eventos executáveis pelo agente em malha aberta. O controlador local $C_{l o c}$ é responsável pelas ações de controle referentes aos objetivos independentes de cada agente. $\mathrm{O}$ controlador de interação $C_{\text {inter }}$ é responsável pelas ações de controle que evitam conflitos entre agentes, ou seja, depende da condição de outros agentes.

A arquitetura proposta é ilustrada pela Figura 1, na qual se consideram dois agentes, $i$ e $j$. A explicação mais detalhada do diagrama se dá na sequência.

A linha tracejada presente no diagrama ilustra que os eventos de um agente não são observáveis por outro agente, garantindo a segurança da privacidade de cada agente autônomo. O que cada agente pode observar em relação aos demais agentes são as desabilitações impostas por eles.

\subsection{Agentes}

Alguns trabalhos na literatura exploram soluções específicas para quando há similaridade entre agentes ( $\mathrm{Su}$ and 


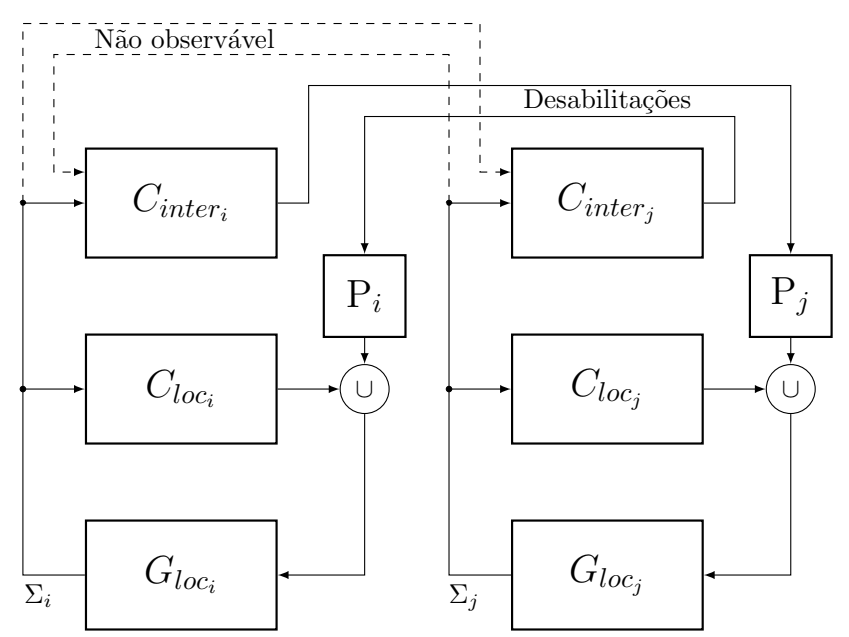

Figura 1. Diagrama ilustrando a arquitetura de controle proposta através de dois agentes $i$ e $j$.

Lin, 2013; Liu et al., 2018, 2019). Essa similaridade é explorada pois é comum que na prática os agentes sejam similares, e.g., em um sistema de enxame de robôs, todos os robôs são idênticos (Lopes et al., 2016). Neste trabalho, os múltiplos agentes são considerados idênticos. Essa consideração torna possível a distribuição do controle sem a necessidade da obtenção de um controlador monolítico. Assim, cada agente pode executar as mesmas funções e ações (eventos). Ainda, essa consideração evidencia os conflitos entre agentes, dado que os conflitos só ocorrem na disputa por tarefas equivalentes. Ainda, considera-se que os agentes são autônomos e não há coordenação nas ações dos agentes como um todo. Nesse caso, é necessário solucionar o problema da disputa por tarefas (ou conflitos), e o problema de bloqueio do sistema. Um exemplo de conflito seria o choque físico entre dois robôs móveis.

Cada agente é um subsistema independente modelado como um SED através de autômatos. O modelo do agente é formado por uma planta local $G_{l o c}$, por um controlador local $C_{l o c}$ e um controlador de interação $C_{\text {inter. }}$ Como todos os agentes são idênticos, então a planta $G_{l o c}$ de cada agente possui estados e eventos equivalentes que representam as mesmas ações executadas por agentes diferentes. Assim o modelo $G_{l o c}$ de cada agente difere dos demais apenas no seu índice de identificação. Cada agente possui uma especificação para o controlador local $C_{l o c}$, que corresponde à sequência de tarefas a serem realizadas. A sequência de tarefas de cada agente pode ser diferente dos demais agentes, dependendo de sua especificação de controle.

O alfabeto da planta de cada agente é determinado como $\Sigma_{i}$, onde $i$ é o índice do agente. Para fins de validação da arquitetura proposta, neste trabalho todos os eventos são considerados controláveis.

\subsection{Controle de Interação}

O controlador de interação $C_{\text {inter }}$ presente em cada agente rege as interações entre agentes. $\mathrm{Na}$ entrada desse controlador são observados apenas os eventos do próprio agente. Através da saída desse controlador são enviadas as desabilitações apenas para outros agentes que estão em concorrência por alguma tarefa com o próprio agente.
Em relação ao quesito segurança, é importante destacar que quando um agente qualquer $x$ envia as desabilitações para outro agente $y$, este último pode inferir algumas informações de $x$ a partir das desabilitações recebidas. Porém, essas informações provenientes das desabilitações estão sempre contidas em um curto espaço de tempo, relacionadas ao estado atual de $x$.

Se houver apenas um agente em atividade, a operação desse será regida pelo controlador local $C_{l o c}$ e não haverá restrições impostas por nenhum $C_{\text {inter }}$. Ainda, se existirem diversos agentes e esses não disputarem por nenhuma tarefa, a operação desses será regida pelos seus controladores locais. O controlador de interação apenas proíbe eventos alheios que representam conflitos para o próprio agente. Quando um agente impõe desabilitações sobre outros agentes, é possível que existam agentes que estejam impossibilitados de executar qualquer evento, ou seja, estes agentes estariam impossibilitados de alcançar os seus estados marcados (exceto se o estado atual seja marcado). Os agentes impossibilitados de alcançar seus estados marcados estão momentaneamente bloqueados.

Em caso de bloqueio, o controlador local $C_{l o c}$ será recalculado de maneira embarcada, sem intervenção do projetista. É importante destacar que essa proposta é diferente do cálculo online do supervisor como realizado no trabalho de Tatsumoto et al. (2018). O recálculo de $C_{l o c}$ será realizado considerando a especificação de controle para o agente sobre a planta com o alfabeto do agente reduzido dos eventos proibidos pelos controladores de interação envolvidos no bloqueio. Na Subseção 3.3 será detalhado o processo de recálculo do controlador local.

O alfabeto do controle de interação é a união dos alfabetos de todos os agentes, ou seja, $\Sigma=\Sigma_{i} \cup \Sigma_{j} \cup \Sigma_{k} \cdots$. É importante destacar que não haverá a explosão exponencial de estados devido a consideração de igualdade entre as plantas dos agentes. Este fato é explanado no exemplo da Seção 4 .

\subsection{Controle Local}

Tarefas a serem executadas pelo agente são modeladas através da especificação para o controlador local $C_{l o c}$. O controlador local pode ser solucionado no âmbito da TCS no framework de Ramadge and Wonham (1987). O alfabeto do controle local é o mesmo da planta de cada agente, ou seja, o controlador local do agente com índice $i$ terá o alfabeto $\Sigma_{i}$.

A fim de possibilitar que o bloqueio seja resolvido localmente entre os agentes, é necessário que a especificação de controle possa ser alterada a qualquer momento. Isto requer que a síntese de $C_{l o c}$ seja realizada de maneira embarcada no agente. Assim, o controle local é considerado adaptativo.

Com a síntese de $C_{l o c}$ sendo realizada de maneira dinâmica e embarcada, não há necessidade da verificação de bloqueio global na etapa de projeto do controle, ou seja, não há necessidade da síntese de um controlador monolítico para a verificação do bloqueio, pois, se um ou mais agentes estiverem bloqueados (dado que não há coordenador), os próprios agentes envolvidos no bloqueio irão recalcular, independentemente, seus controladores locais. 
Descreve-se aqui o processo de recálculo do supervisor. Os agentes são autônomos, qualquer agente bloqueado pode recalcular o controlador local em qualquer momento. Simplesmente, os agentes recalculam seus controladores indefinidamente criando outras alternativas de controle desbloqueando o sistema. É possível que o sistema continue bloqueado após o recálculo do controlador local de um agente, assim, o recálculo continua a ser realizado pelos agentes envolvidos no bloqueio até que o bloqueio seja resolvido.

A descrição matemática do recálculo do controlador é descrita a seguir. Dado que haja dois agentes bloqueados, um deles irá recalcular seu controlador local a partir da sua planta local com o alfabeto restringido pelo controlador de interação do outro agente envolvido no bloqueio. Ou seja, propõe-se que um novo modelo para a planta seja calculado quando existir bloqueio. O autômato do novo modelo com alfabeto restringido é chamado de $G_{l o c}^{\prime}$. E sua linguagem, para um agente qualquer $i$, é definida como:

$$
\mathcal{L}\left(G_{\text {loc }_{i}}^{\prime}\right)=P_{\text {per }_{i}}\left(\mathcal{L}\left(G_{l_{\text {oc }}}\right)\right),
$$

onde:

$$
\Sigma_{\text {per }}(\chi)=\Sigma_{i}-D_{i}(\chi)
$$

é o alfabeto de eventos permitidos para o agente $i$. Onde $D_{i}$ é a união de todas as desabilitações sobre o agente $i$ :

$$
D_{i}(\chi)=D_{i}^{j}\left(q_{j}\right) \cup D_{i}^{k}\left(q_{k}\right) \cup \cdots
$$

e, finalmente $D_{i}^{j}\left(q_{j}\right)$ é o conjunto de eventos desabilitados pelo agente $j$, no seu estado atual $q_{j}$, sobre o agente $i$.

\section{EXEMPLO}

O exemplo consiste em um sistema de transporte com multiagentes. Os agentes são veículos autônomos que possuem um local de destino como objetivo. A especificação de controle é calculada como a menor rota entre o local de origem e o de destino.

Para facilitar o entendimento do exemplo é considerado um mapa que possui 6 regiões: $\mathbf{A}, \mathbf{B}, \mathbf{C}, \mathbf{D}, \mathbf{E}, \mathbf{F}$, conforme mostrado na Figura 2. Sendo a distância entre regiões igualmente distribuídas a fim de simplificar o cálculo da menor rota. Ainda a fim de facilitar o entendimento, as encruzilhadas possuem no máximo três vias interligadas. As plantas $G_{l o c_{i}}$ e $G_{l o c_{j}}$, mostradas na Figura 3, representam o mapa com todas as possíveis rotas e intersecções para os agentes. Cada estado representa uma região de intersecção no espaço, no sentido de que dois agentes não podem ocupar o mesmo espaço (estado) ao mesmo tempo. A ocupação de um mesmo estado por dois agentes significa uma possível colisão entre eles. Desta forma, dois agentes não podem ter o mesmo estado atual em um mesmo momento, visto que isto causaria um conflito. As transições entre os estados representam uma via simples com dois sentidos, i.e., os agentes podem se mover nos dois sentidos entre estados, mas apenas um por vez. Assim, se o agente $i$ ocupa o estado A e o agente $j$ ocupa o estado B, os eventos $a_{i}$ e $b_{j}$ devem ser proibidos para se evitar o choque entre os agentes na trilha entre os estados A e B.

Inicialmente, a especificação de controle considera a sequência de eventos que representa a rota que é o menor caminho entre o ponto inicial e o ponto de destino, isto é, a string de menor comprimento. Os agentes possuem

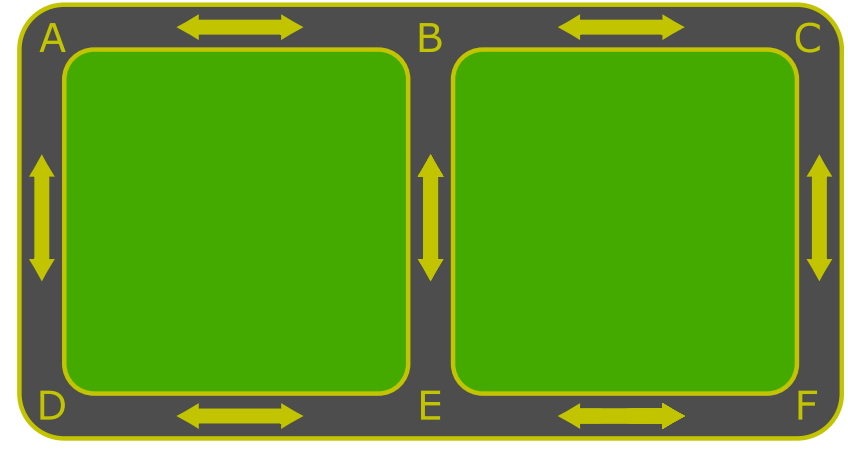

Figura 2. Mapa das regiões e interligações para o sistema de transporte do exemplo.

conhecimento apenas de suas próprias rotas e não existe coordenador, i.e., as possíveis colisões devem ser resolvidas localmente entre os agentes. A especificação para o controle de interação, contida em cada agente, garante que nenhuma colisão irá ocorrer. Entretanto, isto pode gerar um bloqueio. O bloqueio é resolvido pelo recálculo do controle local considerando a especificação do controle de interação. Essa especificação é gerada de maneira embarcada no próprio agente, através de algoritmos de otimização. A síntese dos controladores deve ser feita de maneira embarcada no agente sempre que houver um bloqueio. É importante destacar que a forma de cálculo do controlador é embarcada, porém não é online conforme a técnica utilizada em Tatsumoto et al. (2018), ou seja, somente quando há um bloqueio o controlador completo é recalculado pelo próprio agente.

Neste exemplo são considerados dois agentes, nomeados "agente $i$ " e "agente $j$ ". O agente $i$ está na posição inicial $A$ e sua especificação diz que deve chegar à posição $C$. No caso do agente $j$, a posição inicial é a $C$ e sua especificação diz que deve chegar à posição $A$.

Os controladores locais $C_{l o c_{i}}$ e $C_{l o c_{j}}$ são inicialmente computados como o menor caminho entre os estados iniciais e finais de $G_{l o c_{i}}$ e $G_{l o c_{j}}$, respectivamente. Claramente, é possível perceber que as rotas dos dois agentes irão se cruzar e sem o controle de interação haveria uma colisão entre os agentes. No momento adequado, o controle de interação irá desabilitar eventos, que podem estar habilitados pelo controlador local, para que não haja uma colisão. Esta ação pode gerar um bloqueio no sistema e, se isto ocorrer, o controlador local deverá ser recalculado.

A sequência de eventos se dá como segue. Supõe-se que o agente $i$ execute o primeiro evento $a_{i}$ e assuma a posição $B_{i}$. Como o agente $j$ está ainda na posição inicial $C_{j}$, este está desabilitando a ocorrência dos eventos $c_{i}$ e $k_{i}$. Nesse caso o sistema está bloqueado e é necessário o recálculo do controlador local de um dos agentes. Qualquer um dos agentes poderia recalcular seu controlador local. Considera-se que o agente $j$ recalcula sua rota em $C_{l o c_{j}}$ conforme mostrado na Figura 4.

É possível observar na Figura 4, que o agente $i$ ainda não recalculou o seu controle local. Como o objetivo do agente $i$ é chegar no estado $C_{i}$, é inútil o recálculo do seu controlador local. Assim, quando o agente $j$ executar o evento $l_{j}$, o agente $i$ estará livre para executar o evento $c_{i}$ e finalizar sua tarefa. 


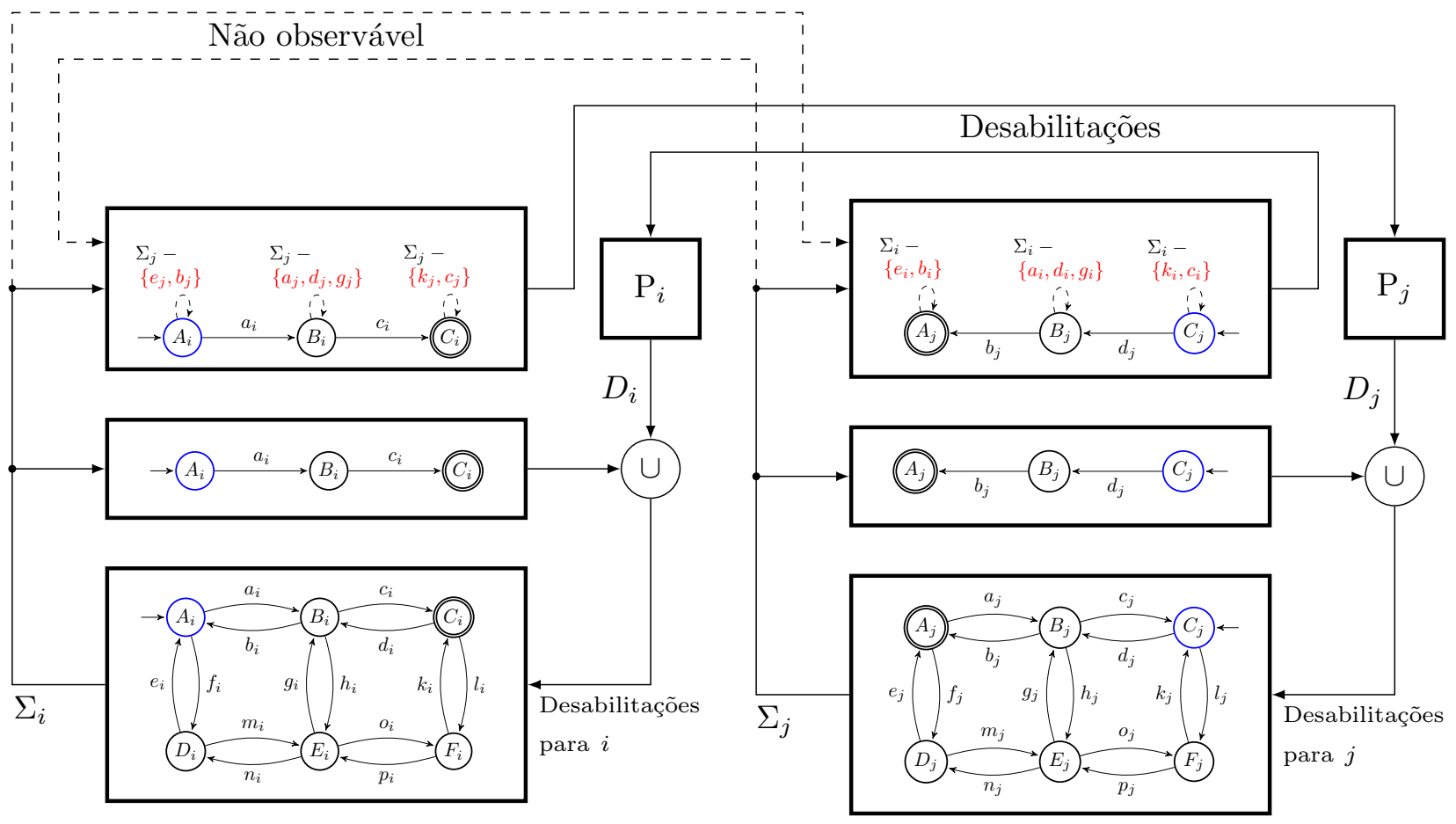

Figura 3. Modelos iniciais para o exemplo. Os estados atuais de cada modelo são mostrados em azul. Os eventos proibidos pelo supervisor de interação são mostrados em vermelho.

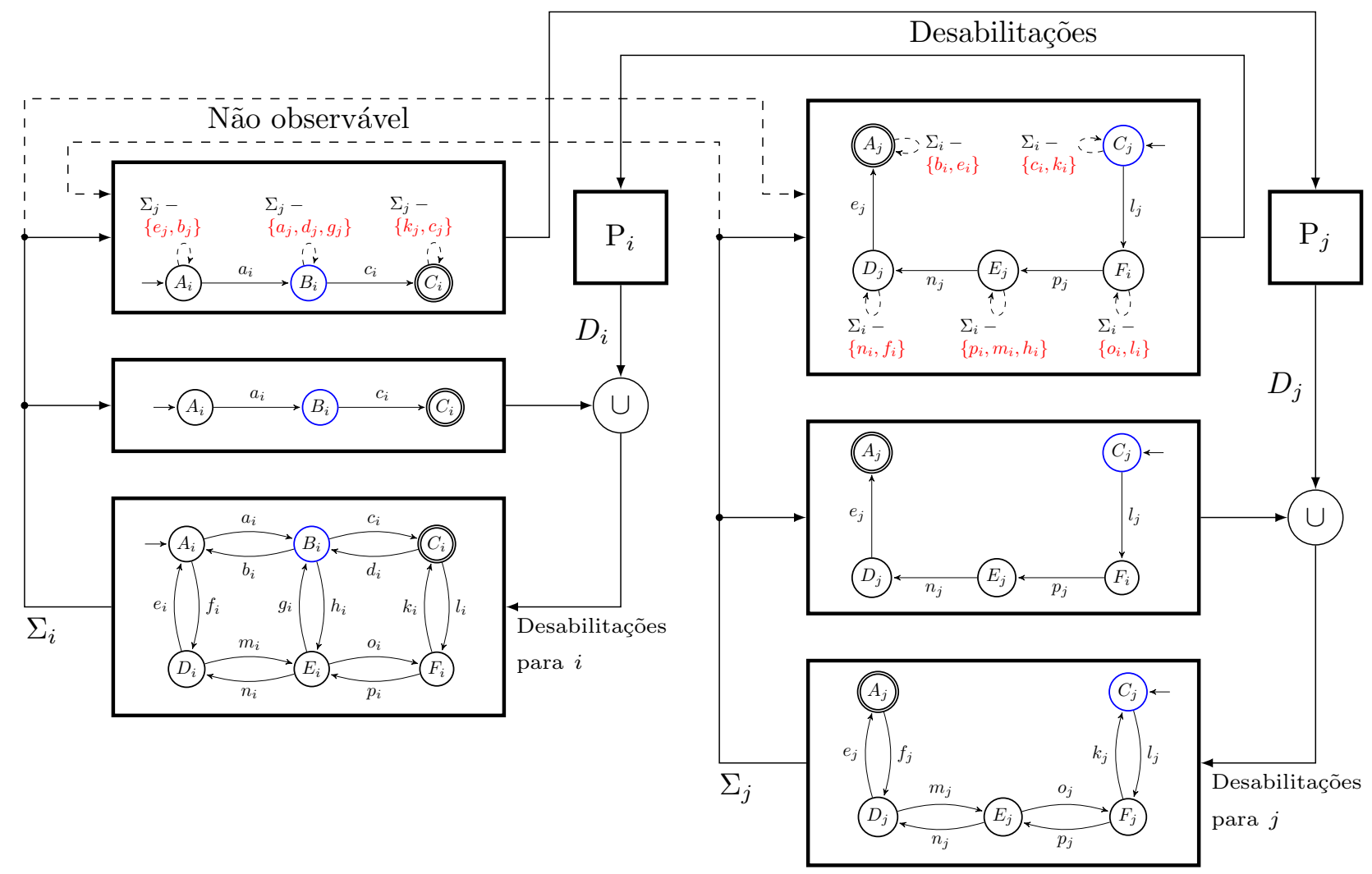

Figura 4. Modelos recalculados após o bloqueio no exemplo. Os estados atuais de cada modelo são mostrados em azul.

Os eventos proibidos pelo supervisor de interação são mostrados em vermelho.

Finalmente, a rota para o agente $j$ é maior que o calculado inicialmente, porém foram resolvidos localmente os problemas de conflito e bloqueio. E, de certa forma, as rotas resultantes ainda foram as menores possíveis considerando os objetivos dos dois agentes. 


\section{CONCLUSÃO \& TRABALHOS FUTUROS}

O trabalho realizado é uma proposta inicial de arquitetura que contempla a solução de dois problemas em aberto na literatura. Um problema solucionado é a necessidade da síntese de um controlador monolítico; e outro problema solucionado é a síntese de controladores para um sistema multiagentes com número variável de agentes no tempo.

Entretanto, ainda há o que desenvolver para que a proposta seja completa. Algumas das considerações devem ser relaxadas para que a solução seja mais generalista. Um dos temas a se desenvolver é a inclusão de eventos não controláveis para contemplar o uso de supervisores conforme a teoria de controle supervisório no contexto de Ramadge and Wonham (1987).

Outro tema a ser desenvolvido é no caso de aplicação para sistemas de transportes. É possível mudar o paradigma de modelagem para autômatos com ponderação de distâncias para o cálculo da otimização de trajetórias (Gonzalez et al., 2018).

A solução proposta neste trabalho considera agentes idênticos, ou seja, com a mesma planta genérica, e desta maneira é possível que um agente desabilite eventos de outros agentes (estes eventos desabilitados são inferidos através da planta do próprio agente). No caso de agentes não idênticos não seria possível realizar esta inferência. Assim se faz necessário verificar a possibilidade do uso da arquitetura proposta com agentes não idênticos, através das propriedades da observabilidade (Lin and Wonham, 1988), observabilidade relativa (Cai et al., 2015) e normalidade (Wang et al., 2018).

Finalmente, é necessário desenvolver os algoritmos para a síntese dos controladores da arquitetura proposta. Estes algoritmos serão agregados à ferramenta Nadzoru (Pinheiro et al., 2015).

\section{AGRADECIMENTOS}

O primeiro autor agradece à CAPES pela bolsa de estudos

\section{REFERÊNCIAS}

Cai, K. and Wonham, W.M. (2010). Supervisor localization: A top-down approach to distributed control of discrete-event systems. IEEE Transactions on Automatic Control, 55(3), 605-618.

Cai, K., Zhang, R., and Wonham, W.M. (2015). Relative observability of discrete-event systems and its supremal sublanguages. IEEE Transactions on Automatic Control, 60(3), 659-670.

de Queiroz, M.H. and Cury, J.E.R. (2000). Modular supervisory control of large scale discrete event systems. Proceedings of the 5th International Workshop on Discrete Event Systems: Analysis and Control, 103-110.

Gonzalez, A.G.C., Alves, M.V.S., Viana, G.S., Carvalho, L.K., and Basilio, J.C. (2018). Supervisory controlbased navigation architecture: A new framework for autonomous robots in industry 4.0 environments. IEEE Transactions on Industrial Informatics, 14(4), 17321743.
Kovalenko, I., Tilbury, D., and Barton, K. (2019). The model-based product agent: A control oriented architecture for intelligent products in multi-agent manufacturing systems. Control Engineering Practice, 86, $105-$ 117.

Lin, F. and Wonham, W. (1988). On observability of discrete-event systems. Information Sciences, 44(3), 173 $-198$.

Liu, Y., Cai, K., and Li, Z. (2018). On scalable supervisory control of multi-agent discrete-event systems. IFACPapersOnLine, 51(7), 25-30.

Liu, Y., Cai, K., and Li, Z. (2019). On scalable supervisory control of multi-agent discrete-event systems. CoRR, abs $/ 1704.08858$.

Lopes, Y.K., Trenkwalder, S.M., Leal, A.B., Dodd, T.J., and Groß, R. (2016). Supervisory control theory applied to swarm robotics. Swarm Intelligence, 10(1), 65-97.

Pinheiro, L.P., Lopes, Y.K., Leal, A.B., and Junior, R.S.U.R. (2015). Nadzoru: A software tool for supervisory control of discrete event systems. IFACPapersOnLine, 48(7), 182 - 187. 5th IFAC International Workshop on Dependable Control of Discrete Systems.

Ramadge, P.J. and Wonham, W.M. (1987). Supervisory control of a class of discrete event processes. SIAM J. Control Optim., 25(1), 206-230.

$\mathrm{Su}, \mathrm{R}$. and Lin, L. (2013). Synthesis of control protocols for multi-agent systems with similar actions. In 52nd IEEE Conference on Decision and Control, 6986-6991.

Tatsumoto, Y., Shiraishi, M., Cai, K., and Lin, Z. (2018). Application of online supervisory control of discreteevent systems to multi-robot warehouse automation. Control Engineering Practice, 81, 97 - 104.

Wang, D., Lin, L., Li, Z., and Wonham, W.M. (2018). State-based control of discrete-event systems under partial observation. IEEE Access, 6, 42084-42093. 